\title{
Survival before and after the introduction of pertuzumab and T-DM1 in HER2-positive advanced breast cancer, a study of the SONABRE Registry
}

\author{
Khava I. E. Ibragimova ${ }^{1,2}$. Sandra M. E. Geurts ${ }^{1,2}$. Sander Croes ${ }^{1}$. Frans Erdkamp ${ }^{3}$. Joan B. Heijns ${ }^{4}$. \\ Jolien Tol ${ }^{5}$ - Birgit E. P. J. Vriens ${ }^{6} \cdot$ Kirsten N. A. Aaldering $^{7} \cdot$ Marcus W. Dercksen $^{8} \cdot$ Manon J. A. E. Pepels $^{9}$. \\ Natascha A. J. B. Peters ${ }^{10}$ - Linda van de Winkel ${ }^{11}$ • Dominique J. P. Tilli ${ }^{1}$. Ingeborg J. H. Vriens ${ }^{1,2} \cdot$ Maaike de Boer ${ }^{1,2}$. \\ Vivianne C. G. Tjan-Heijnen ${ }^{1,2}$ (D)
}

Received: 19 January 2021 / Accepted: 3 March 2021 / Published online: 20 March 2021

(c) The Author(s) 2021

\begin{abstract}
Purpose Immediate and proper implementation of a new and more potent therapy is important to ensure that the patient achieves the best possible outcome. This study aimed to examine whether the real-world overall survival (OS) has improved in patients with human epidermal growth factor receptor 2-positive (HER2+) advanced breast cancer (ABC) since the market release of pertuzumab and T-DM1. Furthermore, we aimed to assess the implementation and survival rates per hormone receptor (HR) subtype.

Patients and methods We included 493 systemically treated patients consecutively diagnosed with HER2 + ABC in 20082017 from the SOutheast Netherlands Advanced BREast cancer (SONABRE) Registry. Median OS was obtained using the Kaplan-Meier method and differences between periods (2008-2012 versus 2013-2017) were tested using multivariable Cox proportional hazards regression modeling. The 3-year implementation rates were estimated for any HER2-targeted therapy, pertuzumab, and T-DM1 by using the competing risk method and calculated from the date of diagnosis of ABC to start of HER2-targeted therapy of interest.

Results The median OS in 2008-2012 versus 2013-2017 was 28.3 versus 39.7 months in all patients (adjusted hazard ratio (adjHR) 0.85, 95\%CI 0.66-1.08), 29.9 versus 36.3 months in patients with HR +/HER2 + disease (adjHR 0.97, 95\%CI 0.72-1.32), and 22.7 versus 40.9 months in patients with HR-/HER2 + disease (adjHR 0.59, 95\%CI 0.38-0.92). Any HER2targeted therapy was used in 79\% of patients in 2008-2012 and in 84\% in 2013-2017. The use of pertuzumab and T-DM1 in 2013-2017 was 48\% and 29\%, respectively. For patients diagnosed with HR +/HER2 + and HR-/HER2 + disease, implementation rates in 2013-2017 were , respectively, 77\% and 99\% for any HER2-targeted therapy, 38\% and 69\% for pertuzumab, and $24 \%$ and $40 \%$ for T-DM1.

Conclusion The survival of patients with HER $2+\mathrm{ABC}$ improved since the introduction of pertuzumab and T-DM1. There is room for improvement in implementation of these HER2-targeted therapies, especially in patients with HR +/HER2 + disease.
\end{abstract}

Keywords Breast neoplasms $\cdot$ Survival $\cdot$ Metastatic breast cancer $\cdot$ HER2-positive disease $\cdot$ Pertuzumab $\cdot$ T-DM1

\section{Introduction}

Approximately one-fifth of patients with advanced breast cancer $(\mathrm{ABC})$ have human epidermal growth factor receptor 2-positive $(\mathrm{HER} 2+)$ disease [1, 2]. Before the introduction

Vivianne C. G. Tjan-Heijnen

vcg.tjan.heijnen@mumc.nl

Extended author information available on the last page of the article of HER2-targeted therapy, patients with HER2 + disease tended to have an aggressive disease course resulting in a poor prognosis [1]. However, after the introduction of trastuzumab in the year 2000, the overall survival (OS) of patients with HER 2 + ABC substantially improved [3-10].

More recently, pertuzumab was approved by the Food and Drug Administration (FDA) in 2012, and the European Medicines Agency (EMA) in 2013, as first-line HER2targeted therapy combined with trastuzumab plus taxane, after the CLEOPATRA trial had shown an impressive 
median OS gain of 16.3 months [11]. Shortly thereafter, the EMILIA trial assessed the efficacy of trastuzumab-emtansine (T-DM1) compared with lapatinib plus capecitabine in patients who were previously treated with trastuzumab and taxane [12]. T-DM1 prolonged median OS significantly by 5.8 months, after which it was implemented for patients with progressive disease on at least one palliative line of trastuzumab-based systemic therapy. Efficacy of T-DM1 was also confirmed in the TH3RESA trial in patients who had received at least two prior palliative HER2-targeted therapies, showing a median survival gain of 6.9 months compared with the treatment of physician's choice [13]. T-DM1 showed comparable survival results as with taxane plus trastuzumab in the first line, whereas the addition of pertuzumab to T-DM1 did not increase the efficacy [14]. Hence, pertuzumab in combination with trastuzumab and taxane became the standard of care as first-line treatment and T-DM1 gained a position as a second and further line of treatment.

To our knowledge, only one real-world study, using the French ESME cohort, looked at survival trends in ABC including the period where pertuzumab and T-DM1 were introduced [9]. They observed steadily improving survival rates in patients diagnosed from 2008 through 2014. The investigators hypothesized that their findings might be related to the market release of new HER2-targeted drugs, although they did not formally test this. Indeed, the impact of pertuzumab and T-DM1 may have been small as during their study these drugs were only just introduced. Conversely, trastuzumab in the early disease setting was implemented very rapidly due to the impressive effectiveness of the drug $[15,16]$. Implementation patterns of new HER2targeted systemic therapies in $\mathrm{ABC}$ are not reported, so far.

The purpose of this study was therefore to examine whether OS has improved in relation to the market release of pertuzumab and T-DM1, by comparing a 5 -year period before (2008-2012) and after (2013-2017) the introduction of these two drugs. Furthermore, we aimed to assess the survival rates and implementation of pertuzumab, and T-DM1 per hormone receptor (HR) subtype, as we have shown before that first-line systemic treatment choices for HER 2 + disease differs by HR status [18].

\section{Patients and methods}

\section{SOutheast Netherlands Advanced BREast cancer (SONABRE) registry}

Data for this study were obtained from the SONABRE Registry (NCT-03577197). This is an ongoing observational cohort study, which aims to include all patients diagnosed with $\mathrm{ABC}$ de novo or during follow-up after early-stage breast cancer from hospitals in the Southeast of the Netherlands. Information, including patient and tumor characteristics, treatment information (surgery, radiotherapy, and systemic treatment, (neo-) adjuvant and palliative), response to systemic therapy, and date, and cause of death, is collected from medical files by specially trained registration clerks. The SONABRE Registry has already been effectively used to perform real-world studies on prognosis, effectiveness, and safety of treatment for ABC $[2,17-20]$. The Medical Research Ethics Committee of Maastricht University Medical Centre approved the registry (15-4-239).

\section{Patients}

We selected patients diagnosed with $\mathrm{ABC}$ in nine hospitals, comprising of one academic, five teachings, and three nonteaching hospitals. Patients were included when diagnosed with HER2 + ABC from January 2008 through December 2017 for eight hospitals, and one hospital from January 2010 through December 2017. Data lock was on February 14, 2020. Patients who received systemic therapy were eligible for analyses, whereas patients not receiving palliative systemic therapy were excluded. HER2 positivity was defined as a positive fluorescence in situ hybridization (FISH) result or an immunohistochemistry score of $3+$. HR (estrogen/progesterone receptor) positivity was defined as positive nuclear staining of $\geq 10 \%$ of one or both receptors. To determine the HR/HER2 status, we used information from the metastatic site $(41 \%)$, and if not available, from a prior recurrence or primary tumor (59\%).

\section{Endpoints and statistical analyses}

Our main objective was to determine whether OS has improved since the introduction of pertuzumab and T-DM1 in 2013 by comparing patients diagnosed with HER $2+$ ABC 5 years before and after the introduction of these new drugs (2008-2012 versus 2013-2017). OS was defined as the time from date of diagnosis of $\mathrm{ABC}$ to date of death or censored at the date of last update. Survival analyses were performed using the Kaplan-Meier method, log-rank tests, and multivariable Cox proportional hazards regression modeling. The prognostic factors included were incidence period, age per year, performance status, HR status, number of initial metastatic sites, initial metastatic sites, and metastatic-free interval (MFI).

The secondary objective was to determine the rate of implementation of any HER2-targeted therapy, pertuzumab, and T-DM1 for the period of 2008-2012 and 2013-2017 and by year of diagnosis. Cumulative use at 3 -year beyond ABC diagnosis of any HER2-targeted therapy, pertuzumab, 
and T-DM1 were assessed by using competing risk analyses. The use of HER2-targeted therapy of interest was defined as 'event' and death without HER2-targeted therapy of interest as 'competing event.' Patients in follow-up were censored at the date of the last update. The number at risk consisted of patients still alive who did not (yet) receive the HER2-targeted therapy of interest. As we expected that HR status might influence the implementation of pertuzumab and T-DM1 in the real world, we additionally looked at the implementation rate, treatment pattern for the first three lines of systemic therapy, and outcome per HR subtype $[8,21$, 22].

The main reason for the non-use of specified HER2-targeted therapy was determined in systemically treated and deceased patients, excluding patients who had died before the market release of pertuzumab (July 30, 2013) and T-DM1 (November 15, 2013).

Baseline characteristics for patients diagnosed in 2008-2012 and 2013-2017 were compared using the chisquare test for categorical variables and Mann-Whitney $U$-test for continuous variables. All reported P-values are two-sided and considered statistically significant at a value of $\leq 0.05$.

\section{Results}

\section{Baseline characteristics per period}

A total of 555 patients diagnosed with HER $2+\mathrm{ABC}$ in 2008-2017 were identified, of whom 493 (89\%) received systemic therapy (Fig. 1). Of these 493 eligible patients, 256 were diagnosed in 2008-2012 and 237 in 2013-2017. Of all patients, $67 \%$ were diagnosed with HR + /HER $2+$ disease and $33 \%$ with $\mathrm{HR}-/ \mathrm{HER} 2+$ disease (Table 1 ). The median follow-up duration was 74 months $(95 \%$ confidence interval (CI) 63-85), during which 352 (71\%) patients had died, and $6(1 \%)$ patients were lost to follow-up due to transfer to a non-participating hospital.

Table 1 shows the baseline characteristics categorized by the period of diagnosis of distant metastases. Overall, patients diagnosed in 2008-2012 tended to be slightly more often diagnosed with cardiovascular comorbidity ( $32 \%$ versus $24 \%, P=0.03$ ), and less often with lymph node and soft tissue metastasis (36\% versus $46 \%, P=0.04$ ), and also less often treated with (neo-) adjuvant endocrine therapy (53\% versus $65 \%, P=0.02$ ) when compared with the more recent period in 2013-2017. When comparing diagnosis periods with regard to HR status, patients with HR +/HER $2+$ disease tended to be older in the more recent period ( $\geq 75$ years, $11 \%$ versus $19 \%, P=0.05$ ), whereas patients with HR-/ HER2 + disease were less frequently diagnosed with visceral metastasis $(77 \%$ versus $59 \%, P=0.01)$ and more often with soft tissue metastasis ( $43 \%$ versus $59 \%, P=0.05$ ) in the more recent period (Supplemental Table S1).

\section{Overall survival}

The median OS of patients diagnosed in 2008-2012 with HER 2 + ABC was 28.3 months (95\% CI 23.4-34.3), as compared with 39.7 months (95\% CI 33.8-49.0) for those diagnosed in 2013-2017 (log-rank $P=0.03$ ), a difference of 11.4 months (Fig. 2a). Among patients with HR +1 HER2 + disease, the median OS was not statistically significant different, 29.9 months (95\% CI 24.5-37.3) for those diagnosed in 2008-2012 and 36.3 months (95\% CI 28.4-47.9) for those diagnosed in 2013-2018 (log-rank $P=0.43$ ) (Fig. 2b). Contrarily, in patients with $\mathrm{HR}-/$ HER 2 + disease, the median OS was 22.7 months (95\% CI 17.7-32.9) when diagnosed in 2008-2012 and 40.9 months (95\% CI 36.4-not reached) when diagnosed in 2013-2017 (log-rank $P=0.005$ ), an improvement in median OS of 18.2 months (Fig. 2c).

In the multivariable model including patients with HER 2 + ABC, the following variables had a significant $(\leq 0.05)$ or borderline significant $(\leq 0.10)$ impact on OS: age at $\mathrm{ABC}$ diagnosis (hazard ratio per year $1.01,95 \% \mathrm{CI}$ 1.01-1.02), WHO performance status $\geq 2$ (hazard ratio 2.29 , 95\% CI 1.58-3.32), WHO performance status unknown (hazard ratio 1.61, 95\% CI 1.27-2.05), multiple initial metastatic sites (hazard ratio 1.68, 95\% CI 1.25-2.25), and MFI 3-23 months (hazard ratio 1.56, 95\% CI 1.12-2.19) (Table 2). The incidence period 2013-2017 was no longer significantly associated with a longer survival time in patients with HER2 + total disease (hazard ratio 0.85, 95\% CI 0.66-1.08). Among patients with HR-/HER2 + disease, incidence period 2013-2017 continued to be associated with a significant longer OS when compared with 2008-2012 (hazard ratio $0.59,95 \% \mathrm{CI} 0.38-0.92$ ).

Supplementary Figure S1 shows the OS per year, categorized by hormone receptor status. The log-rank $P$-value for the trend in OS per year was significant in all patients with HER $2+$ and in patients with HR - /HER 2 + disease ( $P=0.001$ and $P=0.003$, respectively) and borderline significant in patients with HR $+/$ HER $2+$ disease $(P=0.08)$. The 3-year OS rate in all patients with HER2 + disease was 34\% (95\% CI 22-46\%) when diagnosed with $\mathrm{ABC}$ in 2008 and $65 \%$ (95\% CI 46-78\%) when diagnosed in 2017. Patients with HR +/HER $2+$ and HR - /HER2 + disease had a 3-year OS rate of , respectively, 34\% (95\% CI 18-46\%) and $33 \%$ (95\% CI 15-53\%) when diagnosed in 2008, and $56 \%$ (95\% CI $12-31 \%$ ) and $77 \%$ (95\% CI 53-90\%) when diagnosed in 2017. 
Fig. 1 Flow chart study population

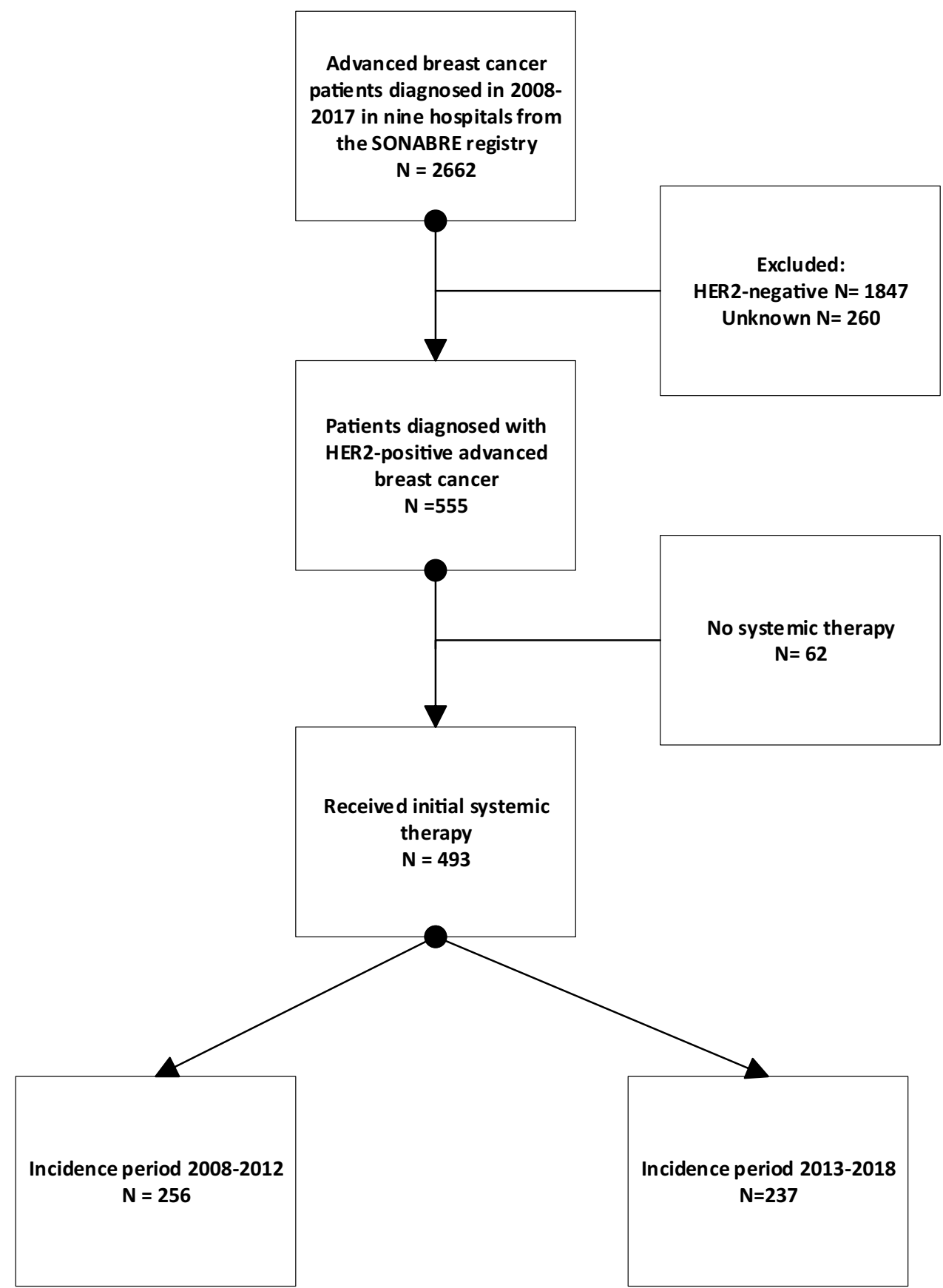

\section{Implementation of HER2-targeted therapy}

Figure 3 and Supplementary Table S2 show the implementation rates for the incidence periods 2008-2012 and 2013-2017, and Supplemental Figure S2 shows the implementation per year. Of systemically treated patients with HER2 + disease, cumulative 3-year use of any HER2-targeted therapy was 79\% (95\% CI 78-88\%) when diagnosed in 2008-2012 and 84\% (95\% CI 78-88\%) when diagnosed in 2013-2017, 3-year use of pertuzumab was $1 \%$ (95\% CI $0.3-3 \%$ ) and $48 \%$ (95\% CI $41-54 \%$ ), and 3-year use of
T-DM1 was 4\% (95\% CI 2-6\%) and 29\% (95\% CI 23-35\%), respectively. For patients with HR +/HER $2+$ disease, the implementation rates in 2008-2012 and 2013-2017 were $73 \%$ (95\% CI $65-79 \%$ ) versus $77 \%$ (95\% CI $70-83 \%$ ) for any HER2-targeted therapy, $0.6 \%$ (95\% CI $0.1-3 \%$ ) versus $38 \%$ (95\% CI 30-45\%) for pertuzumab-based therapy, and $4 \%$ (95\% CI $1-7 \%$ ) versus $24 \%$ (95\% CI $17-31 \%$ ) for T-DM1. For patients with HR-/HER2 + disease, the rates in 2008-2012 and 2013-2017 were 91\% (95\% CI 83-95\%) versus $99 \%$ (95\% CI 91-100\%) for any HER2-targeted therapy, $2 \%$ (95\% CI $0.7-7 \%$ ) versus 69\% (95\% CI 58-78\%) for 
Table 1 Baseline characteristics of systemically treated patients with HER2-positive advanced breast cancer $(\mathrm{ABC})$ in 20082012 versus $2013-2017$

\begin{tabular}{|c|c|c|c|}
\hline & \multicolumn{2}{|c|}{ Period (year of $\mathrm{ABC}$ diagnosis) } & \multirow[t]{2}{*}{$P$} \\
\hline & $2008-2012 N=256$ & $2013-2017 N=237$ & \\
\hline Characteristics & $N(\%)$ & $N(\%)$ & \\
\hline Age at diagnosis $\mathrm{ABC}$ & & & 0.10 \\
\hline$<75$ years & $228(89)$ & $199(84)$ & \\
\hline$\geq 75$ years & $28(11)$ & $38(16)$ & \\
\hline Median (95\% CI) & $60(58-61)$ & $58(58-61)$ & 0.67 \\
\hline \multicolumn{4}{|l|}{ Comorbidity $^{\mathrm{a}}$} \\
\hline Any & $115(45)$ & $102(43)$ & 0.67 \\
\hline Cardiovascular & $83(32)$ & $56(24)$ & 0.03 \\
\hline Diabetes & $30(12)$ & $22(9)$ & 0.38 \\
\hline Lung disease & $23(9)$ & $18(8)$ & 0.58 \\
\hline Cerebrovascular & $10(4)$ & $15(6)$ & 0.22 \\
\hline Non-breast malignancy & $14(6)$ & $16(7)$ & 0.55 \\
\hline WHO performance score & & & 0.30 \\
\hline WHO 0-1 & $124(89)$ & $177(85)$ & \\
\hline $\mathrm{WHO} \geq 2$ & $16(11)$ & $32(15)$ & \\
\hline Missing & 116 & 28 & \\
\hline Hormone receptor status & & & 0.52 \\
\hline $\mathrm{HR}+$ & $168(66)$ & $162(68)$ & \\
\hline $\mathrm{HR}-$ & $88(34)$ & $75(32)$ & \\
\hline Number of initial metastatic site & & & 0.91 \\
\hline Single organ & $106(41)$ & $97(41)$ & \\
\hline Multiple organs & $150(59)$ & $140(59)$ & \\
\hline \multicolumn{4}{|l|}{ Initial metastatic sites ${ }^{\mathrm{a}}$} \\
\hline Bone & $171(67)$ & $148(62)$ & 0.31 \\
\hline Lymph node and soft tissue ${ }^{b}$ & $93(36)$ & $108(46)$ & $0.04 * *$ \\
\hline Visceral $^{\mathrm{c}}$ & $167(65)$ & $146(62)$ & 0.40 \\
\hline $\mathrm{CNS}^{\mathrm{d}}$ & $27(11)$ & $24(10)$ & 0.89 \\
\hline Metastatic-free interval & & & 0.56 \\
\hline$<3$ months/ de novo & 75 (29) & $80(34)$ & \\
\hline 3-23 months & $39(15)$ & $35(15)$ & \\
\hline$\geq 24$ months & $142(56)$ & $122(51)$ & \\
\hline \multicolumn{4}{|l|}{ (Neo-)adjuvant therapy ${ }^{\mathrm{a}, \mathrm{e}}$} \\
\hline Yes & $150(83)$ & $130(83)$ & 0.99 \\
\hline HER2-targeted therapy & $71(39)$ & $72(46)$ & 0.22 \\
\hline Pertuzumab-based therapy & $1(1)$ & $3(2)$ & 0.25 \\
\hline Endocrine therapy & $95(53)$ & $102(65)$ & 0.02 \\
\hline Chemotherapy & $122(67)$ & $100(64)$ & 0.47 \\
\hline No & $31(17)$ & $27(17)$ & \\
\hline
\end{tabular}

$A B C$ advanced breast cancer, $C N S$ central nervous system, $H R$ hormone receptor, $H E R 2$ human epidermal growth factor receptor 2, $\mathrm{WHO}$ World Health Organization

**The observed statistically significant difference may be explained by the difference in definition of soft tissue in the period 2008-2012

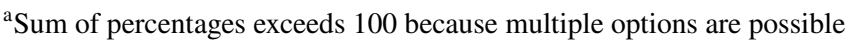

${ }^{\mathrm{b}}$ Lymph nodes, skin, and eye

${ }^{\mathrm{c}}$ Liver, lung, pleura, peritoneum, gastrointestinal track, kidney, adrenal, and ovaries

${ }^{\mathrm{d}}$ Brain and leptomeningeal

${ }^{\mathrm{e}}$ Among patients with recurrent metastases (excluding patients with de novo $\mathrm{ABC}$ ) 

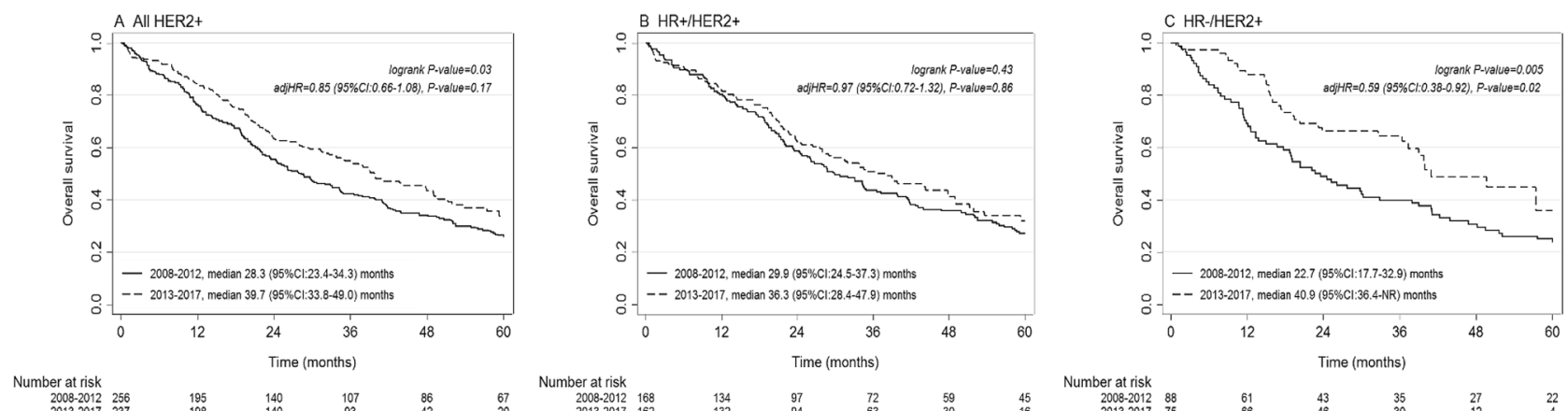

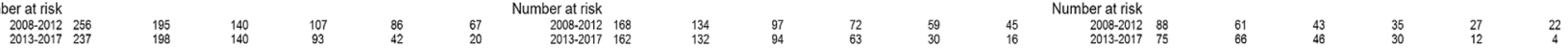

Fig. 2 Overall survival in patients systemically treated for a HER $2+$, b HR +/HER2 +, c HR-/HER2 + ABC by incidence period of ABC diagnosis

Table 2 Multivariable analysis for overall survival (OS) in patients who received at least one line of palliative systemic therapy

\begin{tabular}{|c|c|c|c|c|c|c|c|c|c|}
\hline & \multicolumn{3}{|c|}{$\begin{array}{l}\text { HER2 + total } N=493 \\
\text { events }=352\end{array}$} & \multicolumn{3}{|c|}{$\begin{array}{l}\mathrm{HR}+/ \mathrm{HER} 2+N=330 \\
\text { events }=242\end{array}$} & \multicolumn{3}{|c|}{$\begin{array}{l}\text { HER } 2+\text { total } N=493 \\
\text { events }=352\end{array}$} \\
\hline & Hazard ratio & $95 \%$ CI & $P$ & Hazard ratio & $95 \% \mathrm{CI}$ & $P$ & Hazard ratio & $95 \% \mathrm{CI}$ & $P$ \\
\hline \multicolumn{10}{|l|}{ Incidence period } \\
\hline 2008-2012 & Ref & & & Ref & & & Ref & & \\
\hline 2013-2017 & 0.85 & $0.66-1.08$ & 0.17 & 0.87 & $0.72-1.32$ & 0.86 & 0.59 & $0.38-0.92$ & 0.02 \\
\hline \multicolumn{10}{|l|}{ Age at diagnosis $\mathrm{ABC}$} \\
\hline Age per year & 1.01 & $1.01-1.02$ & 0.002 & 1.02 & $1.01-1.03$ & 0.001 & 1.00 & $0.98-1.02$ & 0.97 \\
\hline \multicolumn{10}{|l|}{ WHO performance status } \\
\hline $0-1$ & Ref & & & Ref & & & Ref & & \\
\hline$\geq 2$ & 2.29 & $1.58-3.32$ & $<0.001$ & 2.89 & $1.83-4.57$ & $<0.001$ & 1.34 & $0.68-2.64$ & 0.40 \\
\hline Unknown & 1.61 & $1.27-2.05$ & $<0.001$ & 1.74 & $1.30-2.33$ & $<0.001$ & 1.48 & $0.95-2.30$ & 0.08 \\
\hline \multicolumn{10}{|l|}{ Hormone receptor } \\
\hline Positive & Ref & & & NA & & & NA & & \\
\hline Negative & 0.86 & $0.68-1.08$ & 0.19 & NA & & & NA & & \\
\hline \multicolumn{10}{|l|}{ Number initial metastatic sites } \\
\hline Single organ & Ref & & & Ref & & & Ref & & \\
\hline Multiple organs & 1.68 & $1.25-2.25$ & 0.001 & 1.46 & $1.02-2.09$ & 0.04 & 2.14 & $1.30-3.53$ & 0.003 \\
\hline \multicolumn{10}{|l|}{ Initial metastatic sites } \\
\hline Bone only & Ref & & & Ref & & & Ref & & \\
\hline Soft tissue without visceral or $\mathrm{CNS}^{\mathrm{a}}$ & 0.80 & $0.49-1.31$ & 0.38 & 0.94 & $0.53-1.65$ & 0.82 & 0.71 & $0.25-2.02$ & 0.52 \\
\hline Visceral without $\mathrm{CNS}^{\mathrm{b}}$ & 1.23 & $0.85-1.78$ & 0.26 & 1.31 & $0.86-2.00$ & 0.21 & 1.27 & $0.55-2.92$ & 0.58 \\
\hline $\mathrm{CNS}^{\mathrm{c}}$ & 1.36 & $0.84-2.20$ & 0.21 & 1.94 & $1.10-3.42$ & 0.02 & 1.06 & $0.38-2.92$ & 0.91 \\
\hline \multicolumn{10}{|l|}{ Metastatic-free interval } \\
\hline$<3$ months/ de novo & Ref & & & Ref & & & Ref & & \\
\hline $3-23$ months & 1.56 & $1.12-2.19$ & 0.009 & 1.84 & $1.20-2.84$ & 0.005 & 1.46 & $0.79-2.70$ & 0.23 \\
\hline$\geq 24$ months & 1.07 & $0.83-1.37$ & 0.61 & 1.14 & $0.84-1.54$ & 0.39 & 0.91 & $0.56-1.47$ & 0.69 \\
\hline
\end{tabular}

$A B C$ advanced breast cancer, $C I$ confidence interval, $C N S$ central nervous system, $H R$ hormone receptor, $H E R 2$ human epidermal growth factor receptor 2, $N A$ not applicable

${ }^{a}$ Lymph nodes, skin and eye

${ }^{\mathrm{b}}$ Liver, lung, pleura, peritoneum, gastrointestinal track, kidney, and ovaries

${ }^{\mathrm{c}}$ Brain and leptomeningeal 

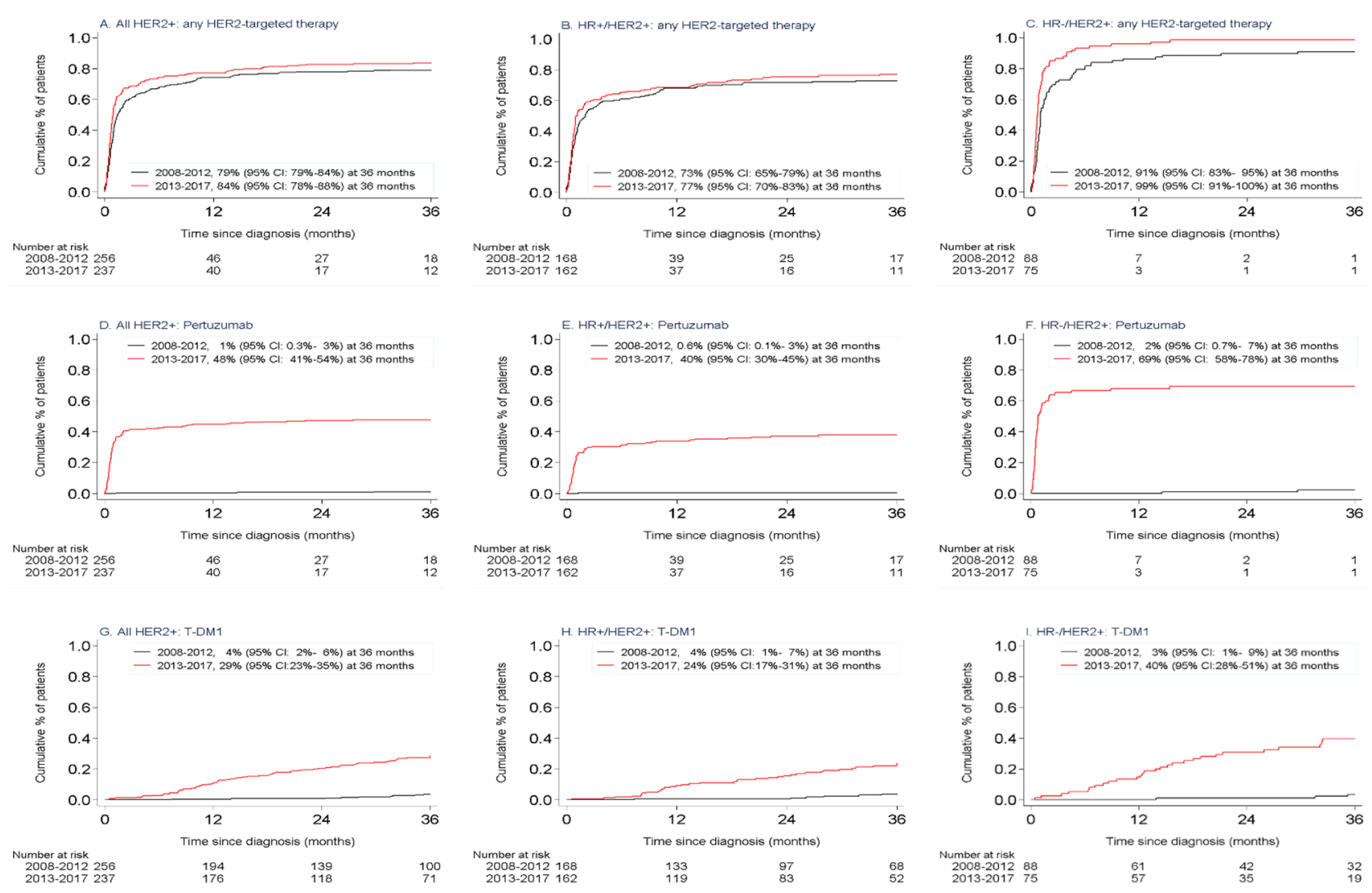

Fig. 3 Use of a-c any HER2-targeted therapy, $\mathbf{d}-\mathbf{f}$ pertuzumab-based therapy, and $\mathbf{g}-\mathbf{i}$ T-DM1 in systemically treated patients and categorized by incidence period and hormone receptor status of

pertuzumab-based therapy, and 3\% (95\% CI 1-9\%) versus $40 \%$ (95\% CI $28-51 \%$ ) for T-DM1, respectively. Among 29 patients who were not treated with HER2-targeted therapy before death, $52 \%$ had a contra-indication for chemo- and/ or HER2-targeted therapy, and for the other $48 \%$, no reason was specified. The reason for non-use was not documented for $70 \%$ of the 75 patients who had not received pertuzumab, and for $76 \%$ of the 17 patients who had not received T-DM1, whereas approximately two-thirds of these had received trastuzumab during their advanced disease course.

Table 3 shows the treatment pattern for the first three lines of systemic therapy categorized by HR status and incidence period. Among patients with HR +/HER2 + disease, the use of HER2-targeted therapy remained similar in lines one through three between the incidence period 2008-2012 and 2013-2017. Around one-third of patients with HR +l HER2 + disease were treated with endocrine monotherapy across all three lines of therapy, irrespective of incidence period. In patients with HR-/HER2 + disease, the use of HER2-targeted therapy increased from approximately $80 \%$ in 2008-2012 to $90 \%$ in 2013-2017.

\section{Discussion}

The value of real-world studies lies in providing insight into the use and effects of new drugs without strict eligibility criteria, thereby helping physicians to interpret and generalize existing data when making treatment decisions [23, 24]. In this ongoing real-world study from the SONABRE registry, we identified 493 systemically treated patients consecutively diagnosed with HER2 + ABC in the period 2008-2017. Here, we present the trend in survival and implementation by comparing the 5-year diagnosis period before (2008-2012) and after (2013-2017) the introduction of pertuzumab and T-DM1. The median OS improved significantly by 11 months in all patients with HER2 + disease. The improvement in OS was particularly evident in the HR-/HER2 + group (median gain of 18 months), while in patients with HR +/HER2 + disease survival remained rather similar. We observed this survival improvement even though the implementation of pertuzumab and T-DM1 was lower than anticipated. Overall, the use of pertuzumab and T-DM1 among patients diagnosed with $\mathrm{ABC}$ in $2013-2017$ was $48 \%$ and $29 \%$, respectively. Implementation of pertuzumab started in the incidence year 2013 at 19\%, increased to $41 \%$ in 2014 and $56 \%$ in 2015 and was constant afterward (61-64\%). The use of T-DM1 was 
Table 3 Treatment pattern, categorized by HR status and incidence period

\begin{tabular}{|c|c|c|c|c|}
\hline \multirow[t]{2}{*}{ HER2 + total } & \multirow[t]{2}{*}{$N$} & \multirow{2}{*}{$\begin{array}{l}\text { HER2-targeted } \\
\%\end{array}$} & \multirow{2}{*}{$\begin{array}{l}\text { ET } \\
\%\end{array}$} & \multirow{2}{*}{$\begin{array}{l}\text { CT } \\
\%\end{array}$} \\
\hline & & & & \\
\hline \multicolumn{5}{|l|}{ 1st line } \\
\hline 2008-2012 & 256 & 62 & 24 & 14 \\
\hline 2013-2017 & 237 & 69 & 27 & 4 \\
\hline \multicolumn{5}{|l|}{ 2nd line } \\
\hline 2008-2012 & 189 & 70 & 21 & 9 \\
\hline 2013-2017 & 164 & 70 & 23 & 7 \\
\hline \multicolumn{5}{|l|}{ 3rd line } \\
\hline 2008-2012 & 134 & 67 & 19 & 15 \\
\hline 2013-2017 & 99 & 73 & 21 & 6 \\
\hline \multicolumn{5}{|l|}{ 1st line } \\
\hline 2008-2012 & 168 & 53 & 36 & 11 \\
\hline 2013-2017 & 162 & 58 & 38 & 4 \\
\hline \multicolumn{5}{|l|}{ 2nd line } \\
\hline 2008-2012 & 134 & 66 & 30 & 4 \\
\hline 2013-2017 & 123 & 64 & 29 & 7 \\
\hline \multicolumn{5}{|l|}{ 3rd line } \\
\hline 2008-2012 & 102 & 61 & 24 & 15 \\
\hline 2013-2017 & 80 & 69 & 25 & 6 \\
\hline \multirow[t]{2}{*}{ HR-/HER2 + } & $N$ & HER2-targeted & $\mathrm{ET}^{\mathrm{a}}$ & $\mathrm{CT}$ \\
\hline & & $\%$ & $\%$ & $\%$ \\
\hline \multicolumn{5}{|l|}{ 1st line } \\
\hline 2008-2012 & 88 & 80 & 1 & 19 \\
\hline 2013-2017 & 75 & 93 & 3 & 4 \\
\hline \multicolumn{5}{|l|}{ 2nd line } \\
\hline 2008-2012 & 55 & 80 & 0 & 20 \\
\hline 2013-2017 & 41 & 90 & 5 & 5 \\
\hline \multicolumn{5}{|l|}{ 3rd line } \\
\hline 2008-2012 & 32 & 81 & 0 & 19 \\
\hline 2013-2017 & 19 & 90 & 5 & 5 \\
\hline
\end{tabular}

$C T$ chemotherapy, ET endocrine therapy, $H R$ hormone receptor, $H E R 2$ human epidermal growth factor receptor

${ }^{\mathrm{a}}$ Includes 3 patients with $\mathrm{HR}+$ primary breast tumor

prominent as of 2013 (26-33\%). One may hypothesize that non-users may have missed out on the survival benefits of pertuzumab or T-DM1.

We observed a median OS of 40 months for patients systemically treated for HER2 + ABC in 2013-2017, which was shorter than the French ESME cohort reporting a median OS of 50 months for the period 2008-2016 [10]. This discrepancy in OS may be caused by differences in screening for $\mathrm{ABC}$ and patient selection. Half of the metastases in the ESME population were found asymptomatically (through screening), whereas screening for distant metastases is not standard practice in the Netherlands. This advancement in diagnosis may explain part of the longer OS observed in the ESME population. In addition, ESME covers 18 Comprehensive Cancer Centers (CCCs), whereas a variety of hospital types are participating in SONABRE. We were not able to analyse the influence of hospital type on survival because the number of patients and hospitals (only one academic hospital) were too small. Nevertheless, one cannot rule out the possibility that the lower use of HER2-targeted therapy plus chemotherapy in the Netherlands also contributes to the lower OS observed in the SONABRE population.

Notably, the survival gain observed in our cohort resembles the results of prior randomized controlled trials $[11,12]$. In the CLEOPATRA trial, the addition of pertuzumab to trastuzumab and docetaxel led to an increase in median OS of 16 months compared with 11 months in our SONABRE cohort [11]. In the EMILIA trial, where T-DM1 was compared with capecitabine/lapatinib, prior use of pertuzumab was not permitted and randomization occurred during the 
disease course making this study difficult to compare with our results [12]. Nevertheless, it is worthy to note that the implementation rate increased each subsequent incidence year and for those diagnosed in 2017, we found a 3-year OS rate of $65 \%$ (95\% CI 46-78\%), equivalent to the 3 -year OS rate of $65.8 \%$ (95\% CI 59.8-71.7\%) observed in the pertuzumab arm of CLEOPATRA. The similarity in outcome is quite surprising. Study patients are generally highly selected, consisting of on average 5\% of the real-world patient population [25]. Indeed, inclusion criteria in the CLEOPATRA consisted of performance score $\leq 1$, no CNS, no uncontrolled medical condition, and an interval of at least 12 months between completion of the (neo-) adjuvant therapy and the diagnosis of $\mathrm{ABC}$, resulting in patients with more favorable baseline characteristics when compared with our real-world population. A similar 3 -year survival rate is even more intriguing when considering the fact that pertuzumab was implemented in only $64 \%$ of the patients diagnosed with ABC in 2017 (Supplemental Figure S2). It remains, however, uncertain how much higher the OS rate would have been with better implementation.

Interestingly, implementation rates and OS differed with regard to HR status. For patients diagnosed with HR + I HER 2 + and HR-/HER 2 + disease, implementation rates in 2013-2017 were, respectively, 38\% and 69\% for pertuzumab and $24 \%$ and $40 \%$ for T-DM1. Even though the implementation rates for pertuzumab and T-DM1 were almost twice as high for patients with HR-/HER2 + disease when compared with $\mathrm{HR}+/ \mathrm{HER} 2$ + disease, they were overall lower than expected. In real life, the implementation rates will never reach $100 \%$ due to the contra-indication for HER2-targeted therapy plus chemotherapy, such as comorbidity (renal dysfunction, cardiovascular disease), performance status $>2$, and patient preferences. Nevertheless, there is still room for improvement, especially in HR +/HER2 + disease where the use of HER2targeted therapy has hardly increased over the years. In the Netherlands, pertuzumab and T-DM1 are fully reimbursed to facilitate the use of these new and expensive agents. However, we are quite conservative in using chemotherapy-based therapy as first-line treatment and prefer endocrine-based therapy for patients with HR + disease and mild symptoms. This could also explain the lower implementation of pertuzumab and T-DM1 found in HR + than in HR-/HER2 ABC and could have led to a missed opportunity to benefit from these new drugs.

The strength of our prospective cohort study lies in the unselected inclusion of all systemically treated patients diagnosed with HER2 + ABC from a ten-year inclusion period in nine different hospitals. The data were manually screened and collected by specially trained registration clerks, which contributed to the high quality of the data. Another strength that sets us apart from other registry studies $[8,9,26,27]$ is that our cohort consists of more recently diagnosed patients with HER2 + ABC, showing a more current treatment pattern, with a substantial long median follow-up duration of
74 months. Our study also has some limitations, inherent to the observational character of the study and by using medical files for the data collection, reasons for non-use are not always clearly documented. A survey study should be conducted on how treatment decisions are made. In addition, the number of patients diagnosed with HR +/HER $2+$ disease is limited $(N=330)$ to observe small OS differences. Nonetheless, this study is an important addition to the evolving demand for real-world studies showing implementation and treatment patterns of new targeted therapies [23, 28]. The reported implementation rate and patient's OS presented in this real-world study were unbiased.

The outcomes presented in this study, providing a realistic implementation pattern, can be utilized for multiple settings, such as budget impact analysis and market penetration estimates of new drugs. Our results also show that more research needs to be done on how treatment decisions are made in daily practice and their subsequent effects on treatment patterns and outcomes. Additionally, physicians need more guidance on how to decide which patients may or may not benefit from new therapies. Previously, a realworld study in stage IV colorectal cancer patients showed that the OS of non-eligible non-trial patients had a significantly worse outcome (HR 1.70, $P<0.01$ ) when compared with trial patients [29]. These results emphasize the need for external validation of trial outcomes. Furthermore, we presented clear differences in survival and implementation rates with regard to HR status. The difference by HR status suggests either a biological impact, an impact of chosen systemic therapies, or differential effectiveness of systemic therapies used. Real-world data on treatment choices and outcomes from an unselected patient population are important to gain more clinical insight into this complex interplay.

\section{Conclusion}

Survival of patients with HER $2+$ ABC improved after the introduction of pertuzumab and T-DM1. The slow and limited implementation of pertuzumab and T-DM1 could have led to a missed survival benefit in some patients. Future studies elucidating the indicators for making systemic treatment choices, especially in HR + /HER 2 + disease, may provide insights into current medical decision-making and assist physicians in making individual treatment choices.

Supplementary Information The online version contains supplementary material available at https://doi.org/10.1007/s10549-021-06178-8.

Acknowledgements We thank our SONABRE registrars of the department of Medical Oncology of Maastricht University Medical Centre (MUMC+), Maastricht, the Netherlands. 
Author contributions KIEI, SMEG, and VCGTH provided study concepts and study design and participated in data analysis and interpretation. .FE, JBH, JT, BEPJV, KNAA, MWD, MJAEP, NAJBP, LW, $\mathrm{MB}, \mathrm{KIEI}$, and DJPT involved in data acquisition. SMEG did quality control of data and algorithms and statistical analysis. .KIEI and SMEG performed manuscript preparation. KIEI, SMEG, SC, FE, JBH, JT, BEPJV, KNAA, MWD, MJAEP, NAJBP, LW, DJPT, IJHV, MB, and VCGTH performed manuscript editing and manuscript review. The International Committee of Medical Journal Editors (ICMJE) criteria for authorship have been met, and we declare that the authors of this manuscript all contributed to the writing of this article.

Funding Data collection was funded by the Netherlands Organization for Health Research and Development (ZonMw: 80-82500-98-8003), Novartis BV, Roche, Pfizer, and Eli Lilly.

Data availability Data are available upon request (vcg.tjan.heijnen@ mumc.nl).

\section{Compliance with ethical standards}

Conflict of interest Khava I.E. Ibragimova Netherlands Organization for Health Research and Development (ZonMw: 80-82500-98-8003); Novartis BV; Roche; Pfizer; Eli Lilly. Sandra M.E. Geurts Netherlands Organization for Health Research and Development (ZonMw: 80-82500-98-8003); Novartis BV; Roche; Pfizer; Eli Lilly. Dominique J.P. Tilli Netherlands Organization for Health Research and Development (ZonMw: 80-82500-98-8003); Novartis BV; Roche; Pfizer; Eli Lilly. Maaike de Boer Netherlands Organization for Health Research and Development (ZonMw: 80-82500-98-8003); Novartis BV; Roche; Pfizer; Eli Lilly. Vivianne C.G. Tjan-HeijnenNetherlands Organization for Health Research and Development (ZonMw: 80-82500-98-8003); Novartis BV; Roche; Pfizer; Eli Lilly. All remaining authors have declared no conflicts of interest.

Ethical approval The Registry was approved and the need for informed consent waived by the Medical Research Ethics Committee of Maastricht University Medical Center (METC 15-4-239).

Open Access This article is licensed under a Creative Commons Attribution 4.0 International License, which permits use, sharing, adaptation, distribution and reproduction in any medium or format, as long as you give appropriate credit to the original author(s) and the source, provide a link to the Creative Commons licence, and indicate if changes were made. The images or other third party material in this article are included in the article's Creative Commons licence, unless indicated otherwise in a credit line to the material. If material is not included in the article's Creative Commons licence and your intended use is not permitted by statutory regulation or exceeds the permitted use, you will need to obtain permission directly from the copyright holder. To view a copy of this licence, visit http://creativecommons.org/licenses/by/4.0/.

\section{References}

1. Slamon D, Clark G, Wong S, Levin W, Ullrich A, McGuire W (1987) Human breast cancer: correlation of relapse and survival with amplification of the HER-2/neu oncogene. Science 235(4785):177-182

2. Lobbezoo DJ, van Kampen RJ, Voogd AC, Dercksen MW, van den Berkmortel F, Smilde TJ et al (2013) Prognosis of metastatic breast cancer subtypes: the hormone receptor/
HER2-positive subtype is associated with the most favorable outcome. Breast Cancer Res Treat 141(3):507-514

3. Slamon DJ, Leyland-Jones B, Shak S, Fuchs H, Paton V, Bajamonde A et al (2001) Use of chemotherapy plus a monoclonal antibody against HER2 for metastatic breast cancer that overexpresses HER2. N Engl J Med 344(11):783-792

4. Marty M, Cognetti F, Maraninchi D, Snyder R, Mauriac L, Tubiana-Hulin M et al (2005) Randomized phase II trial of the efficacy and safety of trastuzumab combined with docetaxel in patients with human epidermal growth factor receptor 2-positive metastatic breast cancer administered as first-line treatment: the M77001 study group. J Clin Oncol 23(19):4265-4274

5. Gasparini G, Gion M, Mariani L, Papaldo P, Crivellari D, Filippelli G et al (2007) Randomized Phase II Trial of weekly paclitaxel alone versus trastuzumab plus weekly paclitaxel as first-line therapy of patients with Her-2 positive advanced breast cancer. Breast Cancer Res Treat 101(3):355-365

6. von Minckwitz G, du Bois A, Schmidt M, Maass N, Cufer T, de Jongh FE et al (2009) Trastuzumab beyond progression in human epidermal growth factor receptor 2-positive advanced breast cancer: a german breast group 26/breast international group 03-05 study. J Clin Oncol 27(12):1999-2006

7. Kaufman PA, Brufsky AM, Mayer M, Rugo HS, Tripathy D, Yood MU et al (2012) Treatment patterns and clinical outcomes in elderly patients with HER2-positive metastatic breast cancer from the registHER observational study. Breast Cancer Res Treat 135(3):875-883

8. Tripathy D, Kaufman PA, Brufsky AM, Mayer M, Yood MU, Yoo B et al (2013) First-line treatment patterns and clinical outcomes in patients with HER2-positive and hormone receptorpositive metastatic breast cancer from registHER. Oncologist 18(5):501-510

9. Gobbini E, Ezzalfani M, Dieras V, Bachelot T, Brain E, Debled $M$ et al (2018) Time trends of overall survival among metastatic breast cancer patients in the real-life ESME cohort. Eur J Cancer 96:17-24

10. Nakano M, Fujisue M, Tashima R, Okumura Y, Nishiyama Y, Ohsako T et al (2015) Survival time according to the year of recurrence and subtype in recurrent breast cancer. Breast 24(5):588-593

11. Swain SM, Miles D, Kim SB, Im YH, Im SA, Semiglazov V et al (2020) Pertuzumab, trastuzumab, and docetaxel for HER2positive metastatic breast cancer (CLEOPATRA): end-of-study results from a double-blind, randomised, placebo-controlled, phase 3 study. Lancet Oncol 21(4):519-530

12. Verma S, Miles D, Gianni L, Krop IE, Welslau M, Baselga $J$ et al (2012) Trastuzumab emtansine for HER2-positive advanced breast cancer. N Engl J Med 367(19):1783-1791

13. Krop IE, Kim SB, Martin AG, LoRusso PM, Ferrero JM, Badovinac-Crnjevic T et al (2017) Trastuzumab emtansine versus treatment of physician's choice in patients with previously treated HER2-positive metastatic breast cancer (TH3RESA): final overall survival results from a randomised open-label phase 3 trial. Lancet Oncol 18(6):743-754

14. Perez EA, Barrios C, Eiermann W, Toi M, Im YH, Conte P et al (2019) Trastuzumab emtansine with or without pertuzumab versus trastuzumab with taxane for human epidermal growth factor receptor 2-positive advanced breast cancer: Final results from MARIANNE. Cancer 125(22):3974-3984

15. Seferina SC, Lobbezoo DJ, de Boer M, Dercksen MW, van den Berkmortel F, van Kampen RJ et al (2015) Real-life use and effectiveness of adjuvant trastuzumab in early breast cancer patients: a study of the southeast netherlands breast cancer consortium. Oncologist 20(8):856-863

16. de Munck L, Schaapveld M, Siesling S, Wesseling J, Voogd AC, Tjan-Heijnen VC et al (2011) Implementation of trastuzumab in 
conjunction with adjuvant chemotherapy in the treatment of nonmetastatic breast cancer in the Netherlands. Breast Cancer Res Treat 129(1):229-233

17. Lobbezoo DJ, van Kampen RJ, Voogd AC, Dercksen MW, van den Berkmortel F, Smilde TJ et al (2016) In real life, one-quarter of patients with hormone receptor-positive metastatic breast cancer receive chemotherapy as initial palliative therapy: a study of the Southeast Netherlands Breast Cancer Consortium. Ann Oncol 27(2):256-262

18. Lobbezoo D, Truin W, Voogd A, Roumen R, Vreugdenhil G, Dercksen MW et al (2016) The role of histological subtype in hormone receptor positive metastatic breast cancer: similar survival but different therapeutic approaches. Oncotarget 7(20):29412-29419

19. van Kampen RJW, Ramaekers BLT, Lobbezoo DJA, de Boer M, Dercksen MW, van den Berkmortel F et al (2017) Real-world and trial-based cost-effectiveness analysis of bevacizumab in HER2negative metastatic breast cancer patients: a study of the Southeast Netherlands Breast Cancer Consortium. Eur J Cancer 79:238-246

20. Lobbezoo DJ, van Kampen RJ, Voogd AC, Dercksen MW, van den Berkmortel F, Smilde TJ et al (2015) Prognosis of metastatic breast cancer: are there differences between patients with de novo and recurrent metastatic breast cancer? Br J Cancer 112(9):1445-1451

21. Cobleigh M, Yardley DA, Brufsky AM, Rugo HS, Swain SM, Kaufman PA et al (2020) Baseline characteristics, treatment patterns, and outcomes in patients with HER2-positive metastatic breast cancer by hormone receptor status from SystHERs. Clin Cancer Res 26:1105

22. Geurts SME, Ibragimova KIE, Teeuwen NJ, Erdkamp F, Vriens BE, den Boer MO et al (2020) Abstract P1-19-34: the role of hormone receptor (HR) status on initial treatment choices and outcomes for human epidermal growth factor receptor (HER)-2 positive advanced breast cancer: a study of the southeast Netherlands advanced breast cancer (SONABRE) registry. Cancer Res. https:// doi.org/10.1158/1538-7445.SABCS19-P1-19-34

23. Zauderer MG (2019) Practical application of real-world evidence in developing cancer therapies. JCO Clin Cancer Inform 3:1-2

24. Stewart M, Norden AD, Dreyer N, Henk HJ, Abernethy AP, Chrischilles E et al (2019) An exploratory analysis of real-world end points for assessing outcomes among immunotherapy-treated patients with advanced non-small-cell lung cancer. JCO Clin Cancer Inform 3:1-15

25. Unger JM, Cook E, Tai E, Bleyer A (2016) The role of clinical trial participation in cancer research: barriers, evidence, and strategies. Am Soc Clin Oncol Educ Book 36:185-198

26. Bonotto M, Gerratana L, Poletto E, Driol P, Giangreco M, Russo $S$ et al (2014) Measures of outcome in metastatic breast cancer: insights from a real-world scenario. Oncologist 19(6):608-615

27. Statler AB, Hobbs BP, Wei W, Gupta A, Blake CN, Nahleh ZA (2019) Real-world treatment patterns and outcomes in HR+/ HER 2+ metastatic breast cancer patients: a national cancer database analysis. Sci Rep 9(1):18126

28. Tsimberidou AM, Ringborg U, Schilsky RL (2013) Strategies to overcome clinical, regulatory, and financial challenges in the implementation of personalized medicine. Am Soc Clin Oncol Educ Book 33:118-125

29. Mol L, Koopman M, van Gils CWM, Ottevanger PB, Punt CJA (2013) Comparison of treatment outcome in metastatic colorectal cancer patients included in a clinical trial versus daily practice in The Netherlands. Acta Oncol 52(5):950-955

Publisher's Note Springer Nature remains neutral with regard to jurisdictional claims in published maps and institutional affiliations.

\section{Authors and Affiliations}

\section{Khava I. E. Ibragimova ${ }^{1,2}$. Sandra M. E. Geurts ${ }^{1,2}$. Sander Croes ${ }^{1}$. Frans Erdkamp ${ }^{3}$. Joan B. Heijns ${ }^{4}$. Jolien Tol ${ }^{5}$ - Birgit E. P. J. Vriens ${ }^{6} \cdot$ Kirsten N. A. Aaldering $^{7} \cdot$ Marcus W. Dercksen $^{8}$ - Manon J. A. E. Pepels ${ }^{9}$. Natascha A. J. B. Peters ${ }^{10}$. Linda van de Winkel ${ }^{11}$ - Dominique J. P. Tilli ${ }^{1}$ Ingeborg J. H. Vriens ${ }^{1,2} \cdot$ Maaike de Boer $^{1,2}$. Vivianne C. G. Tjan-Heijnen ${ }^{1,2} \mathbb{D}$}

1 Department of Medical Oncology, Maastricht University Medical Center, PO BOX 5800, 6202 AZ Maastricht, The Netherlands

2 GROW-School for Oncology and Developmental Biology, Maastricht University, Maastricht, The Netherlands

3 Department of Internal Medicine, Zuyderland Medical Center, Sittard-Geleen, The Netherlands

4 Department of Medical Oncology, Amphia, Breda, The Netherlands

5 Department of Medical Oncology, Jeroen Bosch Hospital, Den Bosch, The Netherlands

6 Department of Internal Medicine, Catharina Hospital, Eindhoven, The Netherlands
7 Department of Internal Medicine, Laurentius Hospital, Roermond, The Netherlands

8 Department of Medical Oncology, Máxima Medical Center, Eindhoven, The Netherlands

9 Department of Internal Medicine, Elkerliek Hospital, Helmond, The Netherlands

10 Department of Internal Medicine, Sint Jans Gasthuis, Weert, The Netherlands

11 Department of Internal Medicine, St Anna Hospital, Geldrop, The Netherlands 